\title{
Studies of Frequency-Dependent Changes under Modulated Ultrasound Exposure on Cells in Suspension
}

\author{
Anna A. Oleshkevich, PhD* \\ Moscow State Academy of Veterinary Medicine and Biotechnology \\ Moscow, the Russian Federation
}

\begin{abstract}
Characteristics of the modulated ultrasound effect (range of modulation frequencies $10 \mathrm{~Hz}-1000 \mathrm{~Hz}$ intensity $0.2 \mathrm{~W} / \mathrm{cm}^{2}$ ) on white blood cells (WBCs) from different animals were studied. The quantitative ratio of WBCs was the most strongly altered by ultrasonic modulation frequency $1000 \mathrm{~Hz}$. This frequency led to degenerative changes of cells. The presence of non-typeable or destroyed cells in smears was indicated. The results obtained demonstrated the possibility of directed impact on different WBC forms.
\end{abstract}

Keywords: ultrasound; modulation; white blood cells.

\section{Introduction}

Studies conducted from the 1960s to the 1990s showed that amplitude-modulated ultrasound (US) used on biological tissue is more efficient than continuous ultrasound [1-4]. This position was confirmed by revealed features of continuous and modulated US influence on testae and blood of different species of animals [5].

The purpose of this study was to detect possible changes in the functional state of WBCs of different species of animals exposed to modulated ultrasound of therapeutic intensity.

\section{Materials and Methods}

In our study, no laboratory animals were harmed. Purposes, methodology and principles of our operations didn't include experiments on living beings. Tests in vivo as well as tests on other animal and human organisms are impractical as long as no satisfactory explanations of phenomena mentioned are found.

The experimental work was carried out at the Department of Information Technology, Mathematics and

*Corresponding author: Anna A. Oleshkevich, Ph.D. Associate Professor, Department of Information Technologies, Mathematics and Physics, Moscow State Academy of Veterinary Medicine and Biotechnology. Mocsow, Russia.E-mail: kompsotita@, gmail.com
Physics of Moscow State Academy of Veterinary Medicine and Biotechnology. All animals were adult and healthy.

Groups of animals: 11 cats aged from 2 to 3 years $(6$ males and 5 females) and 18 horses (an equal number of males and females) aged from 5 to 8 years. Animals' blood was exposed to US with a space-averaged, time-averaged (SATA) intensity of $0.2 \mathrm{~W} / \mathrm{cm}^{2}$ during a period from 15 seconds to 5 minutes, with modulation frequency of $10 \mathrm{~Hz}$ and $1000 \mathrm{~Hz}$ and the carrier frequency of $880 \mathrm{k} \mathrm{Hz}$, according to the previously proven technique [6]. US therapy medical devices were: UST1-01F, UST-5 and UST-1.02S, combined with thermostat $\mathrm{U} 7^{c}$. We applied pulse mode -10 , amplitude modulation pulse ratio - 2, modulator model- GZ-112. We consider it inhumane to receive regularly a large amount of blood from small animals for the extended biophysical series of experiments for reliable statistics. Therefore, the sonication technique has been specifically designed and tested in samples with the minimum volume. We adjusted the US exposure for each blood volume in order to receive comparable results. Blood samples of $1.5 \mathrm{ml}$ (cat) and $10 \mathrm{ml}$ (horse) were sonicated under absolutely identical conditions (oscillator square, cooling fluid, circulative rate). The therapeutic blood cell sonication was carried out in a temperature-controlled cuvette. Its walls were made of US conductive plastic. A coolant, distilled water, circulated continuously (so-called "flow-through cooling").

Blood smears and cytochemical methods

US effects on WBCs were observed under a light microscope. Blood of the same animal, untreated (intact 
specimens) served as a control on determining the US effect on WBCs. Blood smears (control and after the US exposure) were examined under immersion in a transmitted light microscope «Mikmed-5» (optical objective - 100/1.25; ocular lens-10/18).

Smears were made and stained according to the DIFFQUICK technique. Smears were fixed in absolute methanol $15 \mathrm{~s}$, heated in dye solutions for 10 seconds, washed with buffered water, dried and examined under the microscope. WBC count was led via "Meander" line 3-5 along the edges of fields of view smear, 3-5 fields of view at right angles to the middle of the stroke, then 3-5 fields parallel to the edge of the stroke and again at right angles to the edge of the smear. Each sample was examined at least 10 times. We continued our account until 100 whole cells had been estimated [7]. Statistical results processing was performed with the use of "Statistica 6.0" program. Differences were considered significant at $P<0.05$.

\section{Results}

Only extreme ranges of frequency modulation were tested in order to compare and to determine the spectrum of their biological activity in this paper. A detailed comparison of the features of modulated ultrasonic interaction (modulation mode is from 10 to $1000 \mathrm{~Hz}$ ) with blood cells was performed earlier [5]. Preliminarily, we can say that short-term US exposure had multidirectional effects on the WBCs of different animal species. The relative changes in the leukogram were determined after the differential WBC count. The ratio of cells tested to the respective sample control (ctrl) cells was calculated (Table 1.) Some species' features of this interaction were revealed. For example, a 15-second US exposure with modulation of $10 \mathrm{~Hz}$ on cat's blood led to changes in the percentage ratio of cells: the absolute and relative amount of lymphocytes increased 1.5-2 times; the amount of eosinophil and basophil increased 2.5-3 times; the amount of monocytes increased 4 times; band neutrophils, 1.5-2 times, while the amount of segmented granulocytes was reduced $(P<0.05)$. Further increase of exposure time to more than $30 \mathrm{sec}$ enhanced the effect. We observed cell lysis (Photo: Fig.1) and damage of cytoplasmic membrane (CPM).

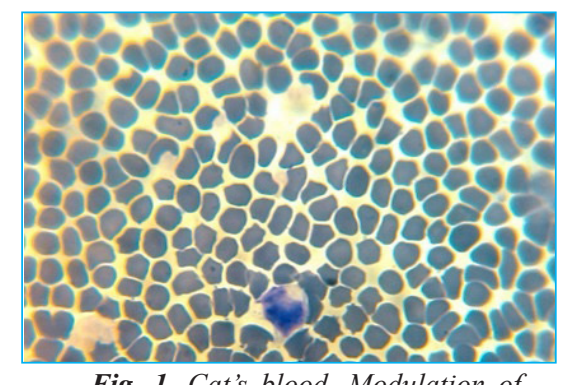

Fig. 1. Cat's blood. Modulation of $10 \mathrm{~Hz}$ for $30 \mathrm{sec}$. Lymphocyte lysis.

Eosinophils and basophils of a horse were not so "sensitive" to short-term exposure: their amount did not change significantly. Monocytes increased 4-5 times compared to the control, $P<0.05$.

Table 1. Effect of the modulated ultrasound with intensity of $0.2 \mathrm{~W} / \mathrm{cm}^{2}$ on WBCs

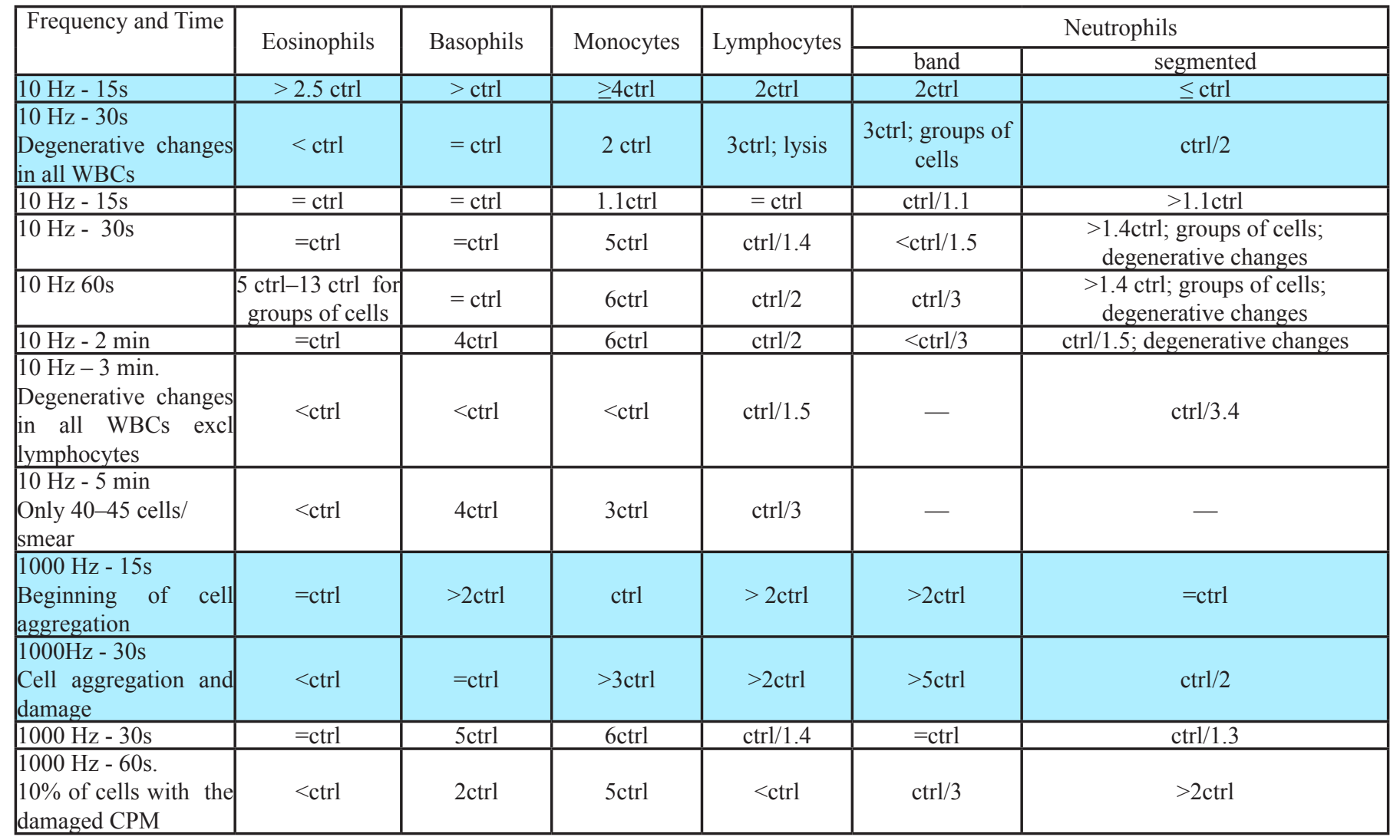

Note: - Cats. - Horses. 
The percentage composition of other formed elements dramatically changed, with the reversed sign, compared with the same effect in cats: the amount of lymphocytes decreased and the amount of segmented neutrophils increased the same -1.4 times against the control, $P<0.05$. Microscopy data showed degenerative changes of segmented neutrophils. Consistent increase in exposure time up to 3 minutes enhanced the effect. Only 40-45 cells were revealed after five-minute exposure (Table 1.): eosinophils, basophils, monocytes and a small number of lymphocytes.

It should be noted that degenerative changes were evidenced in all WBCs of both types of animals (except lymphocytes) (Photo: Fig. 2-5).

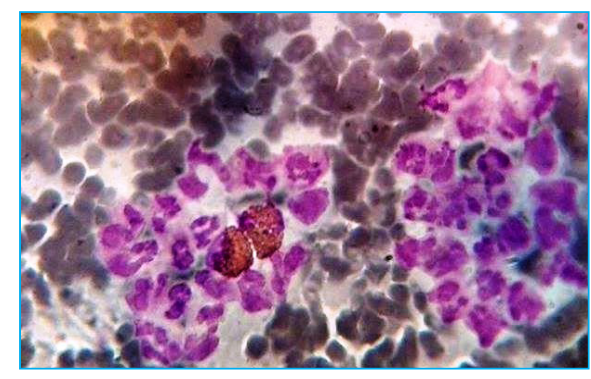

Fig.2. Horse's blood. WBC aggregation at US intensity of $0.2 \mathrm{~W} / \mathrm{cm}^{2}$. Modulation of $10 \mathrm{~Hz}$ for $60 \mathrm{sec}$. The ratio of neutrophils and other types of $W B C s>80 \%$.

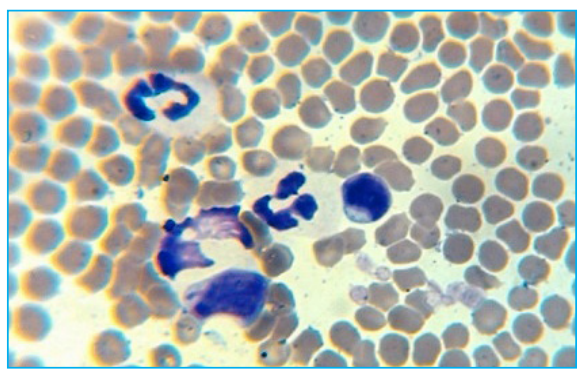

Fig.3. Horse's blood. Modulation of $10 \mathrm{~Hz}$ for $3 \mathrm{~min}$. The segment is seen at the "11 o'clock"position. Group: average sized lymphocyte, segmented neutrophil, monocyte? (butterfly), degenerative leukocyte with destruction, probably, it is the deformed lymphocyte or basophil without cytoplasm (identification is impossible).

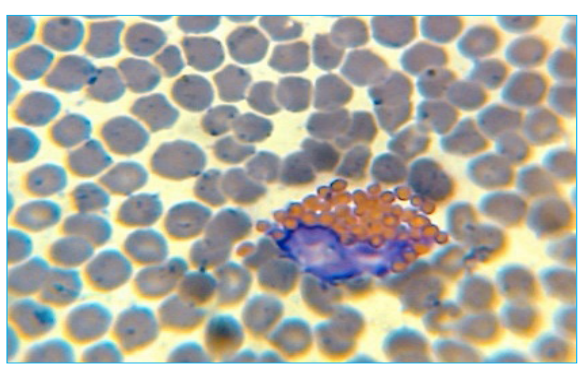

Fig.4. Horse's blood. Modulation of $1000 \mathrm{~Hz}$ for $60 \mathrm{sec}$. Eosinophil. Cell wall destruction, deformation (lysis) of the nucleus. Eosinophilic granules (bottom left) came out of the cell, but were not destructed; nucleus has been torn.

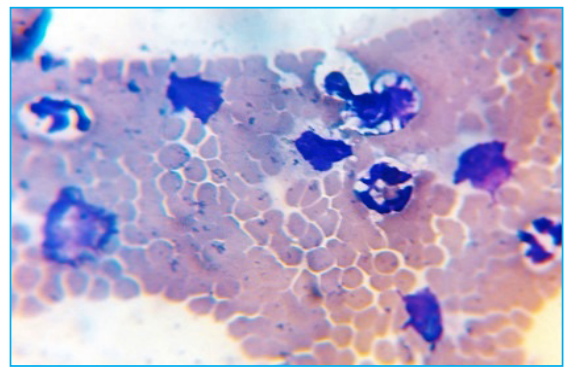

Fig.5. Horse's blood. Modulation of $1000 \mathrm{~Hz}$ for 60 sec. Lysis of the cell membrane and nucleus and, may be, an alteration in surface tension of WBCs of various types. Upper row: segmented neutrophils, lymphocyte, "vacuole" $=2$ cells, connected by nuclei.

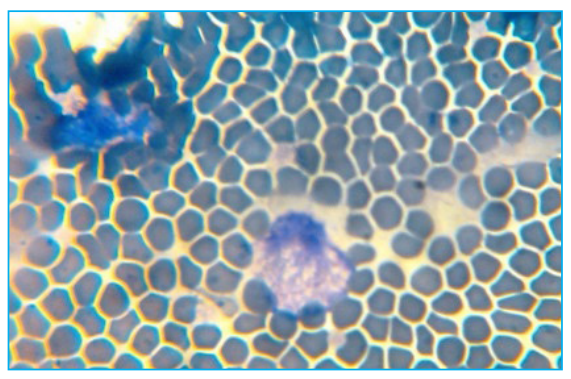

Fig.6. Cat's blood. Modulation of $1000 \mathrm{~Hz}$ for $40 \mathrm{sec}$. Lysis of the WBC.

Effect of modulated US exposure with a frequency of $\underline{1000 \mathrm{~Hz}}$

US waves have the same effect on the eosinophils and basophils of cats and horses: the amount of eosinophil does not change, but the amount of basophil gradually increases (from 2 to 5 times) with increasing of exposure time (1530 seconds) compared to the control. The percentage of lymphocytes and band neutrophils in the leukogram of cats increased 2-5 times; the percentage of segmented neutrophils was reduced 2 times, $P<0.05$. Further increase in exposure time led to cell lysis (Photo: Fig.6) and destruction of the both CPMs and the nuclei of WBCs. The effect on WBCs of a horse was different again. The amount of segmented neutrophils grew (1.3-2 times, $P<0.05)$, while the amount of lymphocytes and band neutrophils decreased; $10 \%$ of the typeable cells had the destructed CPM (Photos: Fig.4, 5).

\section{Discussion}

An analogical consistent pattern-cell aggregation, their partial death, left shift in leukogram, i.e. increase in the amount of young neutrophil in the leukocyte formula (as now we've obtained in cats' blood samples), monocytosis and slight eosinophilia - we also observed under the action of other US intensity $1.0 \mathrm{~W} / \mathrm{cm}^{2}$ with modulation frequency of $1000 \mathrm{~Hz}$ in preliminary studies. The phenomenon of WBC aggregation can be explained by loss of the surface charge of cells [5]. The data obtained from the literature on the effect of continuous and modulated acoustic waves on the cell suspensions were scarce and not very informative. Therefore, our further study of the possibility of non-invasive management of cell 
functional state with the help of ultrasonic waves was relevant and theoretically meaningful.

It was shown earlier that low-intensity US $0.05-0.1 \mathrm{~W} /$ $\mathrm{cm}^{2}$, during the exposure time of 1-3 minutes, had almost no effect on the subsequent growth and development of bacterial culture A. fischeri strain 6 . The impact of $0.4 \mathrm{~W} / \mathrm{cm}^{2}$ had a stimulating effect on bioluminescence and the rate of growth of those bacteria. At intensities over $0.6 \mathrm{~W} / \mathrm{cm}^{2}$ there was an irreversible inhibition of bioluminescence and the number of viable cells progressively decreased [8]. The effect of US on the proliferative rate of transplantable cell culture MDBK was also investigated. The maximum stimulating effect was identified under intensity of $0.05 \mathrm{~W} / \mathrm{cm}^{2}$, exposure $10 \mathrm{sec}$ [9]. Higher intensity of irradiation $\left(0.1 \mathrm{~W} / \mathrm{cm}^{2}\right.$, continue waves) resulted in a significant reduction in cell growth and complete cell destruction (over $0.2 \mathrm{~W} / \mathrm{cm}^{2}$ ). The morphology of cells changed significantly. Plasmolemma was destroyed and the nuclear membrane was ruptured due to the occurrence of microflows inside cells that accompanied the US cavitations in biological media [10]. According to our theory, cell changes in blood of cats and horses were caused by the same factors, but this requires much further investigation and evidence.

Our next objects were WBCs. The influence of US waves and the output of practically important metabolites were studied. A 3-minute US exposure stimulated the release of interferon [11]. There was no cell disruption after treatment with US intensity from 0.01 to $0.05 \mathrm{~W} / \mathrm{cm}^{2}$, the intensity exposure of $0.1 \mathrm{~W} / \mathrm{cm}^{2}$ resulted in decreased viability of WBCs $(-10.6 \%)$. After treatment with US intensity $0.2-2.0 \mathrm{~W} / \mathrm{cm}^{2}$, the number of viable cells decreased even more significantly. Higher US intensity caused a sharp reduction in the viability of WBCs in the suspension due to the threshold of cavitations which, depends on the cell concentration in the bulk medium, and in diluted suspensions coincides with the threshold of cavitations in water $\sim 0.3 \mathrm{~W} / \mathrm{cm}^{2}$. After US influence, the morphology changes in WBCs and their aggregation were found, identified and studied. It was determined that the number of agglomerates formed during a 5-minute US exposure and increased with US intensity [12]. Our last results (modulated US, intensity $0.2 \mathrm{~W} / \mathrm{cm}^{2}$ ) confirmed the 'dose-effect' pattern: cell viability decreased with exposure increase. Photos (Fig. 1-6) show the possible cause of this phenomenon - partial or total CPM damage.

These results made it possible to test the acoustic waves targeting exposure effect on the functional state of WBCs. Experiments on blood of healthy animals in vitro showed stable changes in the state of granulocytes (basophils, eosinophils, neutrophils) and agranulocytes (lymphocytes, monocytes) . The effect depends not only on ultrasound exposure, but also on the inherent characteristics of the species' blood. The mechanism of the biological activity of physical agents is caused by the external field forced vibrations of ions near the surface of the CPM, fluctuations in the channels, and the ability of accelerated ions to penetrate the membrane pores and specialized ion channels due to the active transport systems [13]. It was shown that the modulated UHF, EMR or US effect at some frequencies can cause a change in enzymatic activity towards both activation and inhibition $[14,15]$.

\section{Conclusions}

In brief, we can point out some conclusions:

- Experiments on blood of healthy animals in vitro have shown stable changes in the state of granulocytes and agranulocytes; these experiments have also shown that the effect depends on US exposure, modulation frequency, and on the inherent characteristics of the species' blood.

- Pulse amplitude modulation allowed performance of equal energy exposure on cells and to determine biologically active frequencies.

- Biologically active modulation frequencies $-10 \mathrm{~Hz}$ and $1000 \mathrm{~Hz}$, leading to evidential changes in leukograms of animals, were determined.

- 15-second US exposure with modulation of $10 \mathrm{~Hz}$ on cat's blood led to the absolute and relative increase in the amount of lymphocytes, eosinophils, basophils, monocytes, and band neutrophils.

- 15-second US exposure with modulation of $10 \mathrm{~Hz}$ on horse's blood led to an increase in the amount of monocytes and segmented neutrophils while the amount of lymphocytes decreased.

- Modulated US with a frequency of $1000 \mathrm{~Hz}$ had the same effect on eosinophils (without change) and basophils (gradually increased with increasing of exposure time) of cats and horses. Percentage of lymphocytes and band neutrophils in leukogram of cats increased; the percentage of segmented neutrophils was reduced. In the horse blood the amount of segmented neutrophils grew, while the amount of lymphocytes and band neutrophils decreased.

- Further increase in exposure time led to cell lysis and destruction of the both CPMs and the nuclei of WBCs. Cell viability decreased with exposure increase: 'dose-effect' pattern.

\section{Acknowledgments}

I would like to express my sincere gratitude to veterinarians Komarova E. and Gordiyenko M. for the blood samples provision during the experiments.

\section{References}

1. Andrianov V. The biological effect of ultrasound waves and ultra high frequency electromagnetic oscillations. Morphological changes in the testes caused by ultrasound exposure. Kiev: "Naukova dumka"; 1964. [Book in Russian]. 2. Hwang JH, Tu J, Brayman AA, Matula TJ, Crum LA. Correlation between inertial cavitations dose and endothelial cell damage in vivo. Ultrasound Med Biol 2006; 32(10):1611-9. 3. O'Brien WD Jr, Brady JK, Dunn F. Morphological changes to mouse testicular tissue from in vivo ultrasonic irradiation (preliminary report). Ultrasound Med Biol 1979; 5(1):35-43.

4. Sarvazyan AP, Beloussov LV, Petropavlovskaya MN, Ostroumova TV. The action of low-intensity pulsed ultrasound on amphibian embryonic tissues. Ultrasound Med Biol 1982; $8(6): 639-54$.

5. Oleshkevich A, Pashovkin T. Quantitative analysis of the 
action of the modulated ultrasound on some cells of animal tissues. Veterinary, Animal Science and Biotechnology: Scientific and practical journal 2014; 5:27-33. [Article in Russian].

6. Oleshkevich A, Kuzmina T, Komarova E. Blood cells state analysis after exposure to acoustic (ultrasound) waves. Proceedings of the Conf. "Theoretical and Applied Problems of Education and Science». Tambov 2014; 12:137-138. [Article in Russian].

7. Kondrakhin IP, Kurilov NV, Malakhov AG Clinical laboratory diagnostics in veterinary medicine. M.: Agropromizdat, 1985:59-64. [Article in Russian].

8. Oleshkevich A, Nosovskiy A, Kaminskaya E. Experimental and theoretical basis of enhanced production of cells different etiology after its treatment with acoustic (ultrasonic) waves. Part 1: The method of intensification of metabolic processes of the bacterial cells in suspension. J Biomed Radioeng Radiotech 2014; 2:53-7. [Article in Russian].

9. Oleshkevich A, Nosovskiy A, Kaminskaya E. Experimental and theoretical basis of enhanced production of cells different etiology after its treatment with acoustic (ultrasonic) waves. Part 2: Method of the acoustic stimulation of animal origin cells. J Biomed Radioeng Radiotech 2014; 3:33-9. [Article in Russian].

10. Vivino AA, Boraker DK, Miller D, Nyborg W. Stable cavitation at low ultrasonic intensities induces cell death and inhibits $3 \mathrm{H}-\mathrm{TdR}$ incorporation by Con-A-stimulated murine lymphocytes in vitro. Ultrasound Med Biol 1985; 11(5):751-9 11. Oleshkevich A, Nosovskiy A, Kaminskaya E. Experimental and theoretical basis of enhanced production of cells different etiology after its treatment with acoustic (ultrasonic) waves. Part 4: The experimental justification of the increase of a suspension biosynthesis production, J Biomed Radioeng Radiotech 2014; 11:45-50. [Article in Russian].

12. Oleshkevich A, Nosovskiy A, Kaminskaya E. Experimental and theoretical basis of enhanced production of cells different etiology after its treatment with acoustic (ultrasonic) waves. Part 3: Comparative analysis of methods for assessment of the functional cell state after insonation. $\mathrm{J}$ Biomed Radioeng Radiotech 2014; 8:45-9. [Article in Russian]. 13. Panagopoulos DJ, Karabarbounis A, Margaritis LH. Mechanism for action of electromagnetic fields on cells. Biochem Biophys Res Commun 2002; 298(1):95-102.

14. Uteshev V, Pashovkin T, Gakhova E. Survival of amphibian embryos after amplitude-modulated ultrasound treatment. Bull Mod Med Techn 2010; 4:7-10. [Article in Russian].

15. Uteshev VK, Pashovkin TN, Sevirov AN, Mel'nikova EV, Sadikova DG, Karnaukhov VN. Survival of amphibian embryos after continuous ultrasound treatment. Biofizika 2006; 51(3):539-44. [Article in Russian]. 